\title{
PROCESSO DE ENSINO-APRENDIZAGEM PARA IMPLEMENTAÇÃO DO DIAGNÓSTICO DE ENFERMAGEM EM UNIDADE DE TERAPIA INTENSIVA*
}

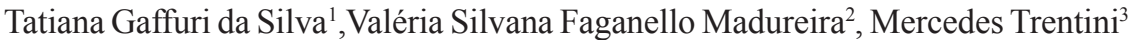

\begin{abstract}
RESUMO: Neste estudo, o propósito foi desenvolver um processo de ensino-aprendizagem teórico-prático com estudantes de graduação sobre o diagnóstico de enfermagem para o cuidado em Terapia Intensiva, captando as experiências dos participantes desse processo. Participaram do estudo 20 estudantes do curso de graduação em Enfermagem regularmente matriculados na disciplina de Enfermagem em unidade de terapia intensiva. O processo foi desenvolvido em três momentos distintos. No primeiro momento, foram realizadas aulas extra-curriculares sobre sistematização da assistência de enfermagem com ênfase no diagnóstico de enfermagem. No segundo, o referencial teórico adotado foi implementado na UTI (campo de estágio da disciplina em questão) e, no terceiro momento, foram realizadas entrevistas semi- estruturadas. Os dados foram analisados através da técnica do Discurso do Sujeito Coletivo. Os discursos evidenciaram mudanças na percepção e no modo de fazer o cuidado de enfermagem, além de práticas centradas na saúde e no ser humano. Com o uso do diagnóstico de enfermagem, o cuidado passa a ser percebido como um momento de aproximação entre cuidador e ser cuidado e o processo a ser valorizado como norteador da assistência.
\end{abstract}

PALAVRAS-CHAVE: Diagnóstico de enfermagem; Cuidados intensivos; Cuidados de enfermagem; Processo ensino-aprendizagem.

\section{LEARNING AND TEACHING PROCESS FOR IMPLEMENTATION OF NURSING DIAGNOSIS IN AN INTENSIVE CARE UNIT}

\begin{abstract}
The study is a part of the leading author's Master's Degree dissertation, which objectified to carry out a teaching and learning process - theoretical as well as practical - with undergraduate students on nursing diagnosis for ICUs, apprehending participants' experiences in this process. Twenty students from the nursing graduation course, regularly enrolled in the subject of Intensive Care Unit, took part in this study. The process was developed in three different steps. First of all, extra-curricular classes were attended on nursing care systematization, emphasis on nursing diagnosis. In the second step, the adopted theoretical background was implemented in the Intensive Care Unit and as the third step, semi-structured interviews were performed. The data were analyzed through the collective subject speech technique. The speeches evidenced changes in the perception and in the way of applying nursing care, besides practices focused on health and human being. By using nursing diagnosis, care is perceived as the closest moment between caregiver and cared individual, and the process is highly valued as a caring reference.
\end{abstract}

KEYWORDS: Nursing diagnosis; Intensive care; Nursing care; Teaching and learning process.

\section{PROCESO DE ENSEÑANZA YAPRENDIZAJE PARA IMPLEMENTACIÓN DEL DIAGNÓSTICO DE ENFERMERÍA EN CENTRO DE TERAPIA INTENSIVA}

RESUMEN: El estudio es un recorte de la disertación de maestría de la autora principal que tuvo el propósito de desarrollar un proceso de enseñanza-aprendizaje, teórico y práctico, con estudiantes graduados sobre el diagnóstico de Enfermería para el cuidado en terapia intensiva, captando las experiencias de los participantes de ese proceso. Participaron del estudio 20 estudiantes del curso de graduación en Enfermería, regularmente matriculados en la asignatura de Enfermería, en la unidad de terapia intensiva. El proceso se ha desarrollado en tres momentos: fueron realizadas clases extracurriculares, sobre sistematización de la asistencia de enfermería con énfasis en el diagnóstico de enfermería. En el segundo momento, el referencial teórico adoptado fue implementado en el CTI (campo de pasantía de la disciplina en cuestión) y, en el tercer momento, fueron realizadas entrevistas semiestructuradas. Los datos fueron analizados a través de la técnica del discurso del sujeto colectivo. Los discursos evidenciaron cambios en la percepción y en la manera de hacer el cuidado de la enfermería, además de prácticas centradas en la salud y en el ser humano. Con el uso del diagnóstico de enfermería, el cuidado pasa a ser percibido como un momento de aproximación entre cuidador y cuidado, y el proceso pasa a ser valorado como referencia de la asistencia.

PALABRAS CLAVE: Diagnóstico de enfermería; Cuidados intensivos; Atención de enfermería; Proceso enseñanza y aprendizaje.

\footnotetext{
*Estudo a partir da Dissertação de Mestrado.

${ }^{1}$ Enfermeira. Mestre em Ciências da Saúde Humana. Professora do Curso de Enfermagem da Universidade do Contestado-UnC Concórdia

${ }^{2}$ Enfermeira. Doutora em Enfermagem. Professora da UnC Concórdia. Orientadora.

${ }^{3}$ Enfermeira. Doutora em Enfermagem.
}

Autor correspondente:

Tatiana Gaffuri da Silva

Rua Victor Sopelsa, 3000 - 89.700-00 - Concórdia-SC Recebido: 23/08/2007

E-mail: tatiana@uncnet.br 


\section{INTRODUÇÃO}

Tentativas de adoção de uma metodologia assistencial de enfermagem vêm ocorrendo nas últimas quatro décadas, como formas de ordenar, planejar e sistematizar as atividades de enfermagem, a fim de obter melhores resultados e o reconhecimento profissional ${ }^{(1)}$.

$\mathrm{O}$ processo de enfermagem, metodologia por meio da qual enfermeiras e enfermeiros podem ordenar e direcionar seu trabalho ${ }^{(2)}$, é definido como atividade intelectual deliberada que aborda a prática de maneira ordenada e sistemática. No Brasil, Horta foi pioneira na proposição de uma metodologia assistencial para a enfermagem, utilizando o método científico como estrutura de organização e sistematização da assistência, num modelo fundamentado na teoria das Necessidades Humanas Básicas ${ }^{(3)}$. Desde então, muitas contribuições já foram feitas ao processo de enfermagem na busca por uma uniformidade de atuação e linguagem; porém, apesar das diversas tentativas, este é ainda pouco utilizado na prática.

Assim, se no discurso, enfermeiras/os defendem a utilização do conhecimento científico e do 'ver o cliente como um todo' como norteadores de sua prática, no cotidiano assistencial a aplicação de uma sistematização que permeie o cuidar é pouco utilizada. Apesar de muitos profissionais perceberem a importância do cuidar sistematizado, acabam reproduzindo o modelo biomédico aprendido durante a graduação e reforçado por aqueles que já atuam na área há algum tempo.

O modelo biomédico é enfatizado em muitas escolas e é possível perceber que estudantes utilizam o processo de enfermagem de forma fragmentada e não reflexiva, sem relacionar os motivos ou razões que provocam as respostas dos clientes a determinadas situações. Isso se reflete na forma com que os mesmos desenvolvem sua prática e desempenham tarefas, deixando de lado a sistematização da assistência, método de trabalho que deveria ser o norteador de sua atuação profissional. Isso tudo sugere repensar a forma como as escolas vêm ensinando a sistematização da assistência a fim de superar o predomínio de práticas biologicistas que têm conduzido à despersonalização do cuidado.

Tal situação tem preocupado os educadores na Enfermagem, levando-os a buscar novos referenciais que permitam ajudar o estudante a desenvolver seu raciocínio, tornando o futuro profissional capaz de identificar as respostas do cliente, da família e da comunidade que comprometem ou poderão comprometer a saúde e o bem-estar dos mesmos, orientando o cuidado para o alcance dos resultados esperados para a saúde.

A possibilidade de mudança de atitude pode ser encontrada no diagnóstico de enfermagem proposto pela North American Nursing Diagnosis Association (NANDA), referencial que exige julgamento das respostas do indivíduo para o seu estabelecimento, possibilitando a definição das intervenções de enfermagem necessárias para o cuidado.

O diagnóstico de enfermagem proposto pela NANDA pode contribuir para uma prática de boa qualidade, uma vez que oferece referência para o planejamento das intervenções de enfermagem e sugere um universo de possibilidades para uma melhor assistência $^{(4)}$.

De acordo com a NANDA ${ }^{(5: 283)}$, o diagnóstico de enfermagem é julgamento clínico sobre as respostas do indivíduo, da família ou da comunidade, a problemas de saúde/processos vitais reais ou potenciais. O diagnóstico de enfermagem proporciona a base para a seleção de intervenções de enfermagem, para atingir resultados pelos quais a enfermeira é responsável.

A razão básica para introduzir o diagnóstico de enfermagem no ensino é o fato de oferecer aos alunos uma forma de processar os dados da avaliação do paciente, estabelecendo relações entre esses dados, de forma a identificar a necessidade ou a demanda de cuidados $^{(6)}$. Na prática, o que se percebe é que, apesar dos professores enfatizarem durante a formação acadêmica a importância do Processo de Enfermagem como norteador e sistematizador da assistência, um grande número de enfermeiras e enfermeiros não o utiliza no cotidiano do exercício profissional.

Da mesma forma, os estudantes, muitas vezes, o consideram mais como um trabalho que deverá ser entregue ao término do ensino teórico-prático do que como um instrumento essencial a ser rotineiramente utilizado como parte integrante e indissociável do cuidado. Além disso, ao utilizá-lo, desenvolvem apenas algumas de suas etapas, centrando a assistência de enfermagem na doença, o que os leva a buscar sinais e sintomas para nortear o cuidado, reduzindo o paciente à sua patologia ou, falando de outro modo, vendo-o como o infarto agudo do miocárdio do leito 1 . O cuidado então, deixa de ver a pessoa para voltar-se à sua doença ou às manifestações desta.

Alguns profissionais tentam utilizar algum referencial norteador para conduzir a sua prática; no

Cogitare Enferm 2007 Jul/Set; 12(3):279-286 
entanto, assim como os estudantes, a maioria deles fragmenta o processo excluindo etapas fundamentais para o assistir. Com isso, minimizam a importância do processo de enfermagem e reduzem a assistência à resolução de problemas, muitas vezes de cunho administrativo, esquecendo de ações pertinentes à Enfermagem, como a busca pelo bem-estar do cliente.

O modelo proposto pela NANDA procura resgatar uma assistência centrada na compreensão da saúde da pessoa, não como um corpo biológico, mas como um todo, um ser individual com desejos, sentimentos e mitos. $\mathrm{O}$ uso do diagnóstico de enfermagem redireciona o cuidar para resolver, minimizar ou fortalecer, em casos positivos, as respostas dos indivíduos aos problemas de saúde e/ou processos vitais ${ }^{(7)}$.

Estas questões, aliadas à certeza da pouca utilização do processo de enfermagem na prática, motivaram o desenvolvimento deste estudo que teve o propósito de desenvolver um processo de ensinoaprendizagem teórico-prático, com estudantes de graduação sobre o diagnóstico de enfermagem para o cuidado em Terapia Intensiva, captando as experiências dos participantes durante o processo.

\section{PROCEDIMENTOS METODOLÓGICOS}

Este estudo identifica-se com a Pesquisa Convergente-Assistencial (PCA) ${ }^{(8)}$, uma vez que se propõe ir ao encontro, através do envolvimento de pesquisador e pesquisados na prática cotidiana, de novas formas de fazer e pensar a assistência/cuidado de enfermagem. A principal característica deste método é a convergência que mantém com a prática, o que permite que pesquisa e prática se mantenham inter-relacionadas. Para que uma pesquisa possa ser considerada como uma Pesquisa Convergente-Assistencial precisa atender a alguns critérios, como segue.

$O$ pesquisador formula o tema de pesquisa a partir de necessidades que emergem de sua prática assistencial: observou-se que os estudantes e profissionais da área, ao cuidarem dos clientes, focalizavam seu interesse nos afazeres do dia-a-dia, tais como a realização de técnicas e o cumprimento de rotinas, esquecendo muitas vezes de considerar o cliente como um todo. Essa problemática vinculada à prática assistencial deu origem a este estudo.

Este método está comprometido com a melhoria direta do contexto social pesquisado: neste sentido, cabe ressaltar que esta pesquisa teve como meta buscar subsídios teóricos que, utilizados e/ ou empregados na prática, possam melhorar o cuidado, bem como subsidiar a atuação de professores no processo ensino-aprendizagem no curso de graduação. Assim, a proposta do estudo está comprometida com os dois campos com os quais mantém interface: o processo de cuidar em UTI e o processo ensinoaprendizagem no curso de graduação em Enfermagem.

O pesquisador assume o papel de provedor de cuidados: durante a coleta de dados, a pesquisadora atuou como provedora de cuidado aos estudantes, no decorrer das aulas teóricas de aprofundamento do estudo do Diagnóstico de Enfermagem e no decorrer das aulas práticas em UTI. Além disso, atuou como cuidadora na assistência de enfermagem desenvolvida com os clientes hospitalizados em UTI, o que fez juntamente com os estudantes.

O espaço físico da pesquisa é aquele onde ocorrem as relações sociais inerentes ao propósito da mesma: a pesquisa foi desenvolvida em espaços físicos intimamente vinculados ao processo ensino-aprendizagem em Enfermagem e ao processo de cuidar em UTI, o que a caracteriza como PCA.

$\mathrm{O}$ estudo foi desenvolvido em duas etapas. A primeira delas deu-se na Universidade do Contestado UnC - Concórdia-SC e a segunda, foi desenvolvida na Unidade de Terapia Intensiva de um hospital geral com 160 leitos. Participaram deste estudo estudantes do curso de graduação em Enfermagem da Universidade do Contestado, que estavam cursando regularmente a disciplina de Enfermagem em Unidade de Terapia Intensiva na sétima fase, oferecida no primeiro semestre de cada ano letivo.

A turma era composta por 45 estudantes e, para o desenvolvimento das aulas teórico-práticas na UTI, o grupo foi subdividido em nove subgrupos com cinco componentes cada, conforme as normas estabelecidas pelo hospital em questão. No desenvolvimento das aulas práticas em UTI, os subgrupos foram acompanhados por duas professoras: uma delas acompanhou seis subgrupos e outra, acompanhou três. Assim, apenas os integrantes dos subgrupos acompanhados pela primeira professora poderiam participar do estudo, o que compôs um total de 30 estudantes. Considerou-se como critério de inclusão a freqüência mínima de $80 \%$ às aulas extracurriculares, o que possibilitou a participação de 20 estudantes.

A parte prática do projeto foi conduzida em três etapas. A primeira constituída pelas aulas extracurriculares. Na segunda, o referencial teórico foi 
implementado na assistência de enfermagem em UTI Adulto e a terceira incluiu as entrevistas realizadas com os estudantes. Na primeira etapa, foram ministradas 60 horas/aula extra-curriculares aos estudantes, abordando a sistematização da assistência através do modelo de processo e de classificação baseado em diferentes autores ${ }^{(3 ; 5 ; 7 ; 9 ; 10)}$.

Na segunda etapa, após o término das aulas, os estudantes aplicaram o conteúdo aprendido na assistência de enfermagem com clientes hospitalizados em UTI. Os estudantes, em grupos de cinco elementos, permaneciam na Unidade por nove dias, totalizando 45 horas. Durante esta fase, o processo de enfermagem era aplicado diariamente, enfatizando o diagnóstico de enfermagem, e ao final de cada dia, professora e estudantes conversavam sobre o processo de enfermagem e sobre os conceitos abordados no marco conceitual, procurando evidenciar as relações existentes entre os mesmos e a prática assistencial.

$\mathrm{Na}$ terceira etapa, foram feitas as entrevistas semi-estruturadas com os estudantes para que pudessem expressar seus pensamentos sobre a experiência vivida. Os dados das entrevistas foram analisados através da técnica do Discurso do Sujeito Coletivo (DSC), proposta de organização e tabulação de dados qualitativos de natureza (neste caso) verbal, que busca dar conta da preservação do pensamento coletivo e extrair de cada um dos depoimentos as expressões-chave e idéias centrais, a fim de compor um ou mais discursos-síntese na primeira pessoa do singular ${ }^{(11)}$.

Para elaborar os DSCs, iniciou-se examinando repetidas vezes cada uma das questões transcritas, procurando selecionar 'partes' relevantes dos textos. Estes trechos, retirados dos discursos, são as expressões chave $(\mathrm{ECH})$, ou seja, partes dos discursos que servem para 'rechear' cada uma das idéias centrais ${ }^{(11)}$.

Após a releitura destes trechos foi possível identificar as idéias centrais, as quais têm a importante função de individualizar os discursos, permitindo a distinção entre um e outro discurso ${ }^{(10)}$. Em seguida, as idéias centrais com sentido semelhante ou complementar foram identificadas e reunidas para dar origem ao discurso síntese.

De acordo com as Diretrizes Nacionais e Internacionais para Pesquisas com Seres Humanos, do Conselho para Organizações Internacionais de Ciências Médicas (CIOMS) e da Resolução No 196/96 do Conselho Nacional de Saúde ${ }^{(12)}$, foram observados os seguintes princípios éticos: as pessoas foram informadas sobre os procedimentos a serem desenvolvidos, thes foi garantida a preservação da privacidade e o respeito à sua vontade de aceitar ou não participar. Dos que atenderam aos critérios de inclusão e que aceitaram participar do mesmo foi requisitada a assinatura do Termo de Consentimento Livre e Esclarecido. O estudo foi aprovado pelo Comitê de Ética em Pesquisa da Universidade do Contestado.

\section{RESULTADOS}

A análise dos dados indicou a existência de expressões-chave relacionadas a três idéias centrais, em torno das quais foram construídos os DSCs. A primeira dessas idéias indica o desenvolvimento do cuidado de Enfermagem anterior à aprendizagem e a utilização do Diagnóstico de Enfermagem, sempre relacionado à patologia, conforme segue.

Idéia Central - Antes, a gente relacionava o cuidado com a patologia.

DSC: Antigamente, a gente só se preocupava com o diagnóstico médico; nós relacionávamos o cuidado com a patologia, com os sinais e sintomas. Tipo, se o paciente era diabético, a gente fazia a prescrição em cima da dieta, só para o diabetes dele. A gente olhava para o paciente, lembrava da patologia e fazia a prescrição. Lá na clínica cirúrgica, por exemplo, a gente via a histerectomia. O mais importante era a cirurgia. A gente se preocupava com a incisão, com o sangramento vaginal (...) pensava mais na técnica do que no envolvimento com o paciente. A gente só via o sistema que estava afetado. A nossa visão era diferente.

O discurso acima evidencia práticas de Enfermagem centradas na doença e nas alterações que ela provoca no corpo: "antigamente a gente só se preocupava com o diagnóstico médico, nós relacionávamos o cuidado com a patologia, com os sinais e sintomas". De forma geral, ao longo dos anos fomos educados a cuidar de sinais e sintomas; não da saúde, como pode nos parecer a um primeiro olhar, mas da doença e de suas manifestações.

Na medicina ocidental, os seres humanos são analisados de um ponto de vista biológico ${ }^{(13)}$. A doença e suas manifestações evidenciam-se muito mais que a saúde e, enquanto a primeira chama a atenção pela sua presença e produz respostas terapêuticas, a saúde não desperta interesse, ficando escondida, sem ganhar importância ${ }^{(14)}$. 
Neste sentido, é pela anomalia que o ser humano se destaca do todo formado pelos homens, e o normal biológico só é revelado pelas infrações à norma ${ }^{(15)}$. Em função disso, compreende-se porque é mais fácil para o estudante cuidar da doença ao invés da saúde.

Nesta situação, a ciência médica teria de ser expressa como a ciência da doença, porque é o estado de doença que, aparecendo, produz um sentimento de perigo e estimula a resposta terapêutica ${ }^{(16)}$. Os profissionais de saúde, contudo, continuam sendo denominados desta forma apesar de, no seu cotidiano, cuidarem muito mais da doença do que da saúde. Desta forma, o que parece haver é uma percepção de saúde como ausência de doença e da doença como algo que se manifesta apenas no corpo.

Razões para tudo isso podem ser encontradas na própria história que, na saúde, é de construções e reconstruções sobre a natureza, as funções, as estruturas do corpo e ainda sobre as relações corpoespírito e pessoa-ambiente ${ }^{(17)}$. As divergências sobre a conceituação de saúde existem desde a época de Hipócrates (460 a 377 a.C.), considerado fonte inspiradora da ciência médica ocidental.

Apesar da redefinição de saúde feita pela Organização Mundial de Saúde (OMS), das outras propostas feitas como 'Saúde para todos até o ano 2000', 'Promoção da saúde' e 'Políticas Públicas Saudáveis' ${ }^{(18)}$ e da mobilização de muitos profissionais para a obtenção daquele 'todo completo' no Ser Humano, na tentativa de estabelecer uma ruptura com a filosofia do modelo anterior, os trabalhadores da saúde, na sua maioria, continuam seguindo um modelo biomédico, tecnicista e operacional que busca a organização do trabalho e que não tem o ser humano completo como preocupação central.

Tal situação é evidenciada no Discurso analisado, no qual fica claro que, embora existam outras formas de ver o processo saúde-doença, em muitos ambientes hospitalares, no cotidiano das ações, o modelo biomédico subsidiado pela noção de saúde como ausência de doença ainda prevalece. Em resumo, na prática o que se percebe é uma busca incessante pela cura da doença, como fica explícito no discurso. Nessa busca, os profissionais procuram identificar sinais e sintomas indesejáveis para a determinação da terapêutica e do cuidar, como se este fosse o único caminho para o alcance da saúde.

Ser saudável depende da capacidade de realização dos planos individuais que cada um determina para a própria vida; implica ter a capacidade e/ou habilidade para satisfazer aqueles objetivos que são necessários e, em conjunto, são suficientes para um mínimo grau de felicidade ${ }^{(19)}$. A maioria das doenças tende a comprometer esta habilidade e podem agir causando dor e sofrimento, porém é possível que não afetem a capacidade da pessoa para satisfazer suas necessidades e para alcançar seus objetivos de vida ${ }^{(19)}$.

Isto quer dizer que uma pessoa pode ter uma doença e ser saudável, como também pode não se sentir saudável apesar de não apresentar nenhuma doença. Daí a importância de utilizarmos, na Enfermagem, uma linguagem e uma forma de sistematização que não veja a doença como o oposto de saúde.

Ser saudável não descarta a possibilidade de doença, de tal forma que saúde e doença não devem ser tratados como fenômenos estranhos, opostos, como têm feito tradicionalmente as ciências da saúde; ao contrário, podem ocorrer simultaneamente, já que não são excludentes ${ }^{(20)}$.

O Diagnóstico de Enfermagem se apresenta como uma proposta que busca romper com concepções estabelecidas sobre o processo saúdedoença, uma vez que não fica restrito à doença e suas manifestações, mas, pelo contrário, faz refletir e ir ao encontro do cliente como um todo.

Os profissionais precisam ampliar o foco e incluir todo o quadro da situação para perceber com igual sensibilidade, não apenas o que se encontra errado, mas também o que está certo ${ }^{(21)}$. É neste sentido que o Diagnóstico de Enfermagem da NANDA avança, como revela o discurso "antigamente... a nossa visão era diferente". Aqui, os estudantes já evidenciam mudanças na concepção de saúde e doença. Quando os mesmos deixam de se preocupar somente com os aspectos biológicos, pressupõe-se que percebam que o bem-estar e o cuidado ao indivíduo vão muito além da simples resolução de sinais e sintomas.

Idéia Central - O diagnóstico de enfermagem favoreceu o cuidado tanto para o paciente como para a família.

DSC: Agora, passamos a cuidar vendo o cliente como um todo. $O$ cuidado melhorou muito e a interação mudou $100 \%$. Estamos escutando mais o que ele tem para dizer, pois conversando ele conta muito, coisas que muitas vezes não tem nada a ver com a doença e, assim, a gente consegue enxergar melhor as necessidades dele. Coisas que antes a gente deixava passar, agora não deixa mais. $O$ 
paciente se sente melhor porque damos mais atenção, conversamos mais e ele percebe que o cuidado não fica restrito, por exemplo, à perna quebrada. Agora não o vemos somente no hospital; vemos na familia, na sociedade, seus sentimentos. Olhamos para ele como ser humano. Trabalhamos também o que está bom, para que se mantenha saudável. A gente consegue dar uma assistência mais ampla, mais humanizada, que prioriza as necessidades dos pacientes. E foi o uso do Diagnóstico de Enfermagem e das outras etapas do processo que permitiram esta troca positiva, que favoreceram o cuidado, tanto para o paciente como para a família.

O DSC acima revela mudanças na percepção dos estudantes frente ao cuidado, após a vivência do Diagnóstico de Enfermagem como referencial para a sistematização da assistência de enfermagem. A prática desta metodologia e as reflexões efetuadas em grupo possibilitaram a aproximação entre estudante e cliente e o início da reconstrução de um cuidar que vai além do corpo biológico, da doença e do hospital, para incluir a vida cotidiana, os sentimentos, a família e a sociedade. Um cuidar que vê e se preocupa com o ser humano presente, priorizando a integralidade do ser no aspecto moral, social, físico e psíquico, ao invés de limitar-se ao indivíduo hospitalizado e ao corpo doente.

Esta forma de pensar e desenvolver o cuidado possibilitou rever aspectos que há muito vêm sendo discutidos, tais como a associação de conhecimento científico e habilidade técnica com interesse, dedicação e sensibilidade tão necessárias a um cuidar humanizado. A junção destes aspectos, além de oferecer segurança ao cuidador e ao ser cuidado, pode romper com a sensação de vazio na relação cliente/profissional, promovendo uma assistência de qualidade.

Este romper com o vazio tem como pressuposto a expressão, por parte dos profissionais, de sentimentos positivos e negativos, tentando desenvolver uma relação de cuidado humano de ajuda e confiança, que implica cultivar empatia, calidez, compatibilidade, além de verdade, honestidade, aceitação e compromisso ${ }^{(22)}$.

O cuidado é, então, percebido como um processo de troca, presença, co-responsabilidade e envolvimento entre cliente e profissional. No entanto, a valorização destes aspectos pelos estudantes só foi possível através da interação com o cliente, necessária para avaliar como os indivíduos, famílias e comunidades respondem aos acontecimentos vividos, informações indispensáveis para a identificação e confirmação dos Diagnósticos de Enfermagem.

Com isso, percebe-se que o Diagnóstico de Enfermagem, como uma das etapas do processo de Enfermagem, enfatiza o julgamento clínico, ou seja, exige a realização de inferências sobre os dados captados e relativos às respostas desencadeadas por problemas de saúde ou por processos de vida que possibilitam o planejamento e realização das intervenções de Enfermagem ${ }^{(23)}$. Nesse sentido, a atividade diagnóstica é um fator possibilitador de maior aproximação entre os enfermeiros e seus clientes, já que requer maior conhecimento das respostas físicas e emocionais destes ${ }^{(24)}$.

Nesta percepção, a visão do todo e o encontro entre os sujeitos se evidenciam como fortalecedores do cuidar, porém, apesar desta concepção, ainda há forte predominância do tecnicismo incorporado ao longo dos anos. Não se trata de negar a importância do desenvolvimento tecnológico para a melhoria da assistência de Enfermagem, nem a importância do conhecimento técnico-científico na orientação do fazer, mas sim de enfatizar que práticas exclusivamente mecanicistas têm levado à despersonalização do cuidado e não conseguem responder a questões que se referem à integralidade e à unicidade do ser humano.

Percebe-se, com isso, que o desafio para estudantes e profissionais é justamente compreender que as habilidades básicas e essenciais para o cuidar ultrapassam o fazer técnico e a habilidade manual, o que sugere o encontro de um ponto de equilíbrio onde, numa escala de valores, a técnica, o fazer e a visão do ser humano como um todo tenham o mesmo significado e valor.

Idéia Central - Para usar o diagnóstico de enfermagem tivemos de aprender diferente do que tínhamos aprendido.

DSC: Quando começamos a usar o diagnóstico de enfermagem, estranhamos, porque tivemos de aprender diferente do que tínhamos aprendido; foi dificil ter uma visão mais ampla, porque a gente se centrava na patologia. Precisava de tempo para aplicar e estudar e no estágio tínhamos de fazer a parte técnica e o processo. Então, no início foi lento, mas depois ficou gostoso. A linguagem usada no DE foi um empecilho, também porque é diferente daquela que a gente usava antes. A gente não tinha o hábito de fazer um bom histórico e foi dificil mudar a forma 
de fazê-lo. Agora a gente se empenha mais, viu a importância que ele tem e levanta coisas sobre todo o organismo da pessoa e sobre a parte psicossocial, psicobiológica e psicoespiritual[...]. Começamos a dar mais valor para o PE, porque afinal é ele que norteia todas as nossas ações. Depois de levantar o histórico, você analisa e identifica os DEs e os FRs. Com essa reflexão, fica fácil prescrever e prestar uma assistência bem mais ampla. Depois, conversando com o cliente, você avalia a assistência e sabe se a prescrição foi eficiente ou não, para então fazer outras e mudar de acordo com as necessidades.

$\mathrm{O}$ fato de os trabalhadores de Enfermagem estarem habituados a uma rotina assistencial prédeterminada, aliada à falta de reflexão sobre o emprego de uma metodologia assistencial no dia-a-dia de trabalho, faz com que o cuidado rotineiro prevaleça e haja um distanciamento entre o cuidado que se quer e o que de fato se faz. Daí a estranheza dos alunos ao se depararem com um modelo que exigia ir além da patologia.

No ensino, esta realidade também se evidencia nas escolas que, na sua maioria, ainda não utilizam este referencial como sistematizador da assistência. Mesmo que alguns professores já estejam adotando o DE nos campos da prática, os conteúdos das disciplinas, na maioria das vezes, não têm sido construídos com base nesse embasamento teórico, o que faz com que os DEs continuem ocupando apenas um espaço nos conteúdos relativos às patologias. Identificar o diagnóstico de enfermagem envolve a ativação de uma série de habilidades mentais que permitem que sejam feitas inferências sobre os dados observáveis ${ }^{(22)}$.

No discurso, os estudantes relatam a dificuldade de "pensar diferente", o que sugere que não estavam habituados a passar por um processo de reflexão e inferência para chegar aos diagnósticos. Aqui, é preciso considerar que a busca pelo novo, o rompimento com o velho, em geral é processo longo e doloroso, pois os seres humanos têm forte tendência à acomodação ${ }^{(25: 334)}$. Assim, transformar conceitos, pontos de vista, modos de cuidar há muito consolidados não é tarefa fácil ou simples, porém é a opção que nos direciona para a efetivação do cuidado holístico e do emprego de uma metodologia assistencial na Enfermagem que valorize o diagnóstico de enfermagem.

\section{CONSIDERAÇÕES FINAIS}

Este estudo revela mudanças na percepção e no modo de fazer dos estudantes sobre cuidado de enfermagem após o uso do diagnóstico de enfermagem. Alguns pontos importantes devem ser considerados, uma vez que mudam a forma com que o estudante, futuro profissional, percebe o cliente e o cuidado de enfermagem.

$\mathrm{Na}$ percepção dos estudantes, as práticas de enfermagem deixam de ser centradas na doença e nas suas manifestações, colocando a saúde como foco do cuidado, o que os leva a valorizar as necessidades dos indivíduos no que se refere ao bem-estar, segurança, alegria, atenção, zelo. O cuidado passa a ser percebido como um momento de troca, de aproximação, que não valoriza exclusivamente os aspectos biológicos e as manifestações da doença. Está patente aqui o reconhecimento da necessidade de conquistar novas capacidades, tais como interesse, amor e comunicação, as quais extrapolam as intelectuais e técnicas.

A mudança de comportamento requer mais que o reconhecimento de aspectos que precisam ser mudados e repensados; exige o engajamento total de todos os envolvidos, escola, estudantes, docentes, serviço de enfermagem e instituição de saúde, para que esta forma de raciocínio [o DE] seja de fato incorporada pelos profissionais.

Percebe-se, com isso, que ainda há um longo caminho a ser percorrido até a utilização do Diagnóstico de Enfermagem na prática de cuidado. Daí, ressaltase a importância de instrumentalizar a equipe de Enfermagem e os docentes, uma vez que este é certamente um caminho para a consolidação de um conhecimento próprio da Enfermagem, que busca melhorar as relações entre profissionais e clientes, ajudar os indivíduos a viver melhor e melhorar a qualidade do cuidado de Enfermagem.

A metodologia utilizada com a realização do curso extra-curricular e as atividades realizadas na Unidade de Terapia Intensiva foi extremamente importante e significativa, pois o método da Pesquisa Convergente Assistencial permitiu a articulação da prática e a pesquisa. A realização de estudos de caso, seguidos de discussão, foi também um ponto positivo que deve ser valorizado, uma vez que se mostrou relevante no momento da efetivação do ensino teóricoprático. Percebemos que, após inúmeros exercícios em sala de aula, os estudantes mostravam-se mais aptos para a construção do Processo de Enfermagem e para a integração do diagnóstico de enfermagem com as demais fases do Processo de Enfermagem.

Cabe ao corpo docente olhar para a prática 
procurando identificar com clareza o que ensina, como ensina e que resultados estão sendo obtidos com os métodos utilizados. É necessário que a escola, em todos os seus momentos e instâncias, tenha consciência de seu papel e de sua responsabilidade frente ao cuidado de Enfermagem, para que possa aceitar o desafio de construir e reconstruir constantemente a prática de Enfermagem.

\section{REFERÊNCIAS}

1 Barros ALBL. Trabalho docente assistencial de enfermagem no Hospital São Paulo da UNIFESP/EPM [tese]. São Paulo (SP): Universidade Federal de São Paulo; 1998.

2 George JB. Teorias de enfermagem. Porto Alegre: Artes Médicas; 2000.

3 Iyer P, Taptich BJ, Bernocchi-Losey D. Processo e diagnóstico em enfermagem. Porto Alegre: Artes Médicas; 1993.

4 Cruz DA. Contribuições do diagnóstico de enfermagem para a autonomia da enfermeira. $2^{\mathrm{a}}$ ed. Brasília: Associação Brasileira de Enfermagem; 1997.

5 North American Nursing Diagnosis Association (NANDA). Diagnósticos de enfermagem: definições e classificações - 2003-2004. Porto Alegre: Artmed; 2005.

6 Cruz DA. Aintrodução do diagnóstico de enfermagem no ensino: sua influência no processamento de informações por alunos de graduação [tese]. São Paulo (SP): Universidade de São Paulo; 1995.

7 Bennedet SA, Bub MBC. Manual de diagnósticos de enfermagem: uma abordagem baseada na teoria das necessidades humanas e na classificação diagnóstica da NANDA. Florianópolis: Bernúcia; 2001.

8 Trentrini M, Paim L. Pesquisa em enfermagem: uma modalidade convergente-assistencial. Florianópolis: UFSC; 1999.

9 Carpenito LJ. Diagnósticos de enfermagem: aplicação à prática clínica. $6^{a}$ ed. Porto Alegre: Artes Médicas; 1997.

10 Carpenito LJ. Manual de diagnósticos de enfermagem. $6^{\mathrm{a}}$ ed. Porto Alegre: Artes Médicas; 1999.

11 Lefèvre F, Lefèvre AC. Discurso do sujeito coletivo: um novo enfoque em pesquisa qualitativa. Caxias do Sul: EDUCS;2003.

12 Brasil. Fundação Nacional de Saúde. Resolução 196/96 sobre pesquisa envolvendo seres humanos. Brasília, 1996.

13 Capra A. Abordagem hermenêutica da relação saúde doença. Cad Saúde Publ. 2003 Jul/Ago;19(4):923-31.

14 Gadamer HG. Verdade e método: traços fundamentais de uma hermenêutica filosófica. $2^{\mathrm{a}}$ ed. Petrópolis: Vozes; 1998.

15 Canguilhem G. O Normal e o patológico. Rio de Janeiro: Forense Universitária; 1995.

16 Coelho MTAD, Almeida Filho N. Conceitos de saúde. Hist Cienc Saude-Manguinhos. 2002 Mai/Jun;9(2):315-33.

17 Albuquerque CMS, Oliveira CPF. Saúde e doença: significações e perspectivas em mudança. 2005 [acesso em 2005 Out]. Disponível em: <http: www.ipv.pt/millenium/ Millenium 25/25_27.htm>.

18 Brasil. Ministério da Saúde. Secretaria de Políticas de saúde. As cartas da promoção da saúde. Brasília, 2002.

19 Nordenfelt L. Conversando sobre saúde: um diálogo filosófico. Florianópolis: Bernúncia; 2000.

20 Madureira VSF. Eu, você - nós: co-partícipes no educar [dissertação]. Florianópolis (SC): Universidade Federal de Santa Catarina; 1994.

21 Remen RN. O paciente como ser humano. São Paulo: Summus; 1993.

22 Lacerda MA. O cuidado transpessoal de enfermagem no contexto domiciliar [dissertação]. Florianópolis (SC): Universidade Federal de Santa Catarina; 1996.

23 Cruz DA. A Inserção do diagnóstico de enfermagem no processo assistencial. In: Cianciarullo TI, Gualda DMR, Melleiro MM, Anabuki MH. Sistema de assistência de enfermagem: evolução e tendências. São Paulo: Ícone; 2001.

24 Braga CG, Cruz D. A taxonomia II proposta pela North American Nursing, Diagnosis Association (NANDA). Rev Latino-AmEnferm. 2003 Mar/Abr;11(2):240-4.

25 Erzinger AR, Trentini M. Enfermeiras e enfermeiros frente aos desafios no início da carreira profissional. Rev Téc Científica Enferm. 2003;1(5):332-9. 